\title{
Avaliação da Cardiotoxicidade induzida por Quimioterapia em pacientes com Câncer de Mama
}

\author{
Evaluation of Chemotherapy-induced Cardiotoxicity in Breast Cancer patients

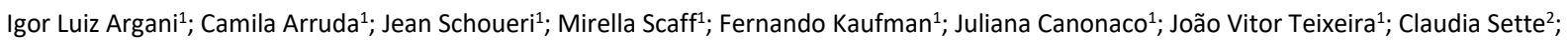 \\ Daniel Cubero²; Auro del Giglio ${ }^{2}$
}

Como citar: Argani IL, Arruda C, Schoueri J, et al. Avaliação da Cardiotoxicidade induzida por Quimioterapia em pacientes com Câncer de Mama. Clin Onc Let. 2018;3(1-2):37-44. https://doi.org/10.4322/col.2018.002

\begin{abstract}
Resumo
Objetivo: Avaliar a alteração da função cardíaca em pacientes com câncer de mama tratadas com quimioterapia. Métodos: Estudo observacional retrospectivo com coleta de dados em prontuário no Hospital Mário Covas (Santo André - Brasil). Avaliou-se a fração de ejeção obtida pelo ecocardiograma (ECO) antes do tratamento quimioterápico, em comparação com o ECO ao final do tratamento. Resultados: Foram incluídas 234 pacientes com câncer de mama submetidas a QT, das quais 100 realizaram ao menos um ECO e 33, pelo menos dois exames. Desse total, apenas 18 puderam ser analisados, pois apresentavam ECO feito corretamente, i.e., antes e depois do tratamento quimioterápico. Analisando esses ECOs, observou-se diferença da fração de ejeção média de $5 \%$ (IC95\% 2,9-7,6; p=0,0001). Conclusão: A alta prevalência e o expressivo aumento da sobrevida de pacientes com câncer de mama indicam a necessidade de acompanhamento adequado dos efeitos a longo prazo da QT. A análise dos ECO feitos adequadamente nos pacientes com câncer de mama nesse estudo demonstrou significante queda da fração de ejeção, confirmando os efeitos cardiotóxicos da QT. Apesar disso, apenas uma fração pouco expressiva dos pacientes apresentou acompanhamento adequado.
\end{abstract}

Palavras-chave: Câncer de Mama, Cardiotoxicidade, Saúde Pública

\begin{abstract}
Objective: Evaluate the alteration of cardiac function in breast cancer patients treated with chemotherapy. Methods: Retrospective observational study with data collection in medical records at Hospital Mário Covas (Santo André - Brazil). The ejection fraction obtained by the echocardiogram (ECO) before the chemotherapy treatment was evaluated, in comparison with the ECO at the end of the treatment. Results: 234 breast cancer patients undergoing chemotherapy were included, of whom 100 performed at least one ECO and 33 at least two exams. Of this total, only 18 could be analyzed, since they had ECO done correctly, i.e., before and after the chemotherapy treatment. Analyzing these ECOs, we observed a difference of the mean ejection fraction of $5 \%(95 \% \mathrm{Cl} 2.9-7.6, p=0.0001)$. Conclusion: The high prevalence and significant increase in survival of breast cancer patients indicate the need for adequate follow-up of the long-term effects of QT. The analysis of echocardiogram performed adequately in patients with breast cancer in this study demonstrated a significant decrease in ejection fraction, confirming the cardiotoxic effects of chemotherapy. Despite this, only a small fraction of the patients presented adequate follow-up.
\end{abstract}

Key-words: Breast cancer; Cardiotoxicity; Public Health

\footnotetext{
1 Estudantes de graduação em medicina da Faculdade de Medicina do ABC, Santo André, SP, Brasil

2 Departamento de oncologia da Faculdade de Medicina do ABC, Brasil. Av. Lauro Gomes, 2000 - Vila Sacadura Cabral, Santo André - SP, $09060 \_870$

Financiamento: CEPHO (Centro de Estudos e Pesquisa em Hematologia e Oncologia)

Conflito de interesse: Todos os autores responsabilizam-se pelo conteúdo do presente estudo e negam conflitos de interesse que possam ter influenciado os resultados deste trabalho.
} 


\section{INTRODUÇÃO}

O câncer de mama trata-se de um importante problema de saúde pública no Brasil, sendo o câncer mais comum entre as mulheres - à exceção do câncer de pele não-melanoma. Segundo o Instituto Nacional de Câncer (INCA), a estimativa de novos casos para o ano de 2016 é de $57.960 .^{1}$

De acordo com os dados coletados pelo Ministério da Saúde, entre 25\%-35\% das pacientes já chegam aos centros especializados em estadios avançados, necessitando de tratamento quimioterápico. ${ }^{2}$ Neste cenário, a cardiotoxicidade se apresenta como importante efeito adverso do tratamento oncológico, sendo responsável por considerável morbimortalidade. ${ }^{3}$

Dentre os principais efeitos lesivos ao sistema cardiovascular, a disfunção sistólica do ventrículo esquerdo levando à insuficiência cardíaca - destaca-se por sua maior frequência e gravidade..$^{3,4}$

Tendo em vista a possível lesão miocárdica secundária ao uso de quimioterápicos, torna-se necessário a monitorização da função cardíaca destes pacientes. O método diagnostico mais utilizado para avaliação dos danos é o ecocardiograma (ECO). Estudos indicam a necessidade de realização de ECO previamente à quimioterapia, especialmente associada ao uso de antraciclinas, havendo necessidade de modificação do tratamento proposto por guidelines - devido a sua cardiotoxicidade - em cerca de 1,3-2,8\% dos pacientes. ${ }^{5,6}$

Dentre os métodos de diagnóstico e acompanhamento das lesões causadas por quimioterápicos, o ecocardiograma é o método mais utilizado, uma vez que apresenta baixo custo, fácil acesso e caráter não invasivo. Além disso, permite avaliar não só a função sistólica, como a função diastólica, as valvas cardíacas e o pericárdio. ${ }^{7,8}$

Considerando o aumento expressivo da sobrevida dos pacientes diagnosticados com neoplasia de mama, e associado a este fato a utilização de quimioterápicos de ação cardiotoxica conhecida, bem como o constante desenvolvimento de novos agentes quimioterápicos, estudos que avaliem os métodos de vigilância e que busquem marcadores precoces de alterações a nível cardíaco em pacientes oncológicos são imprescindíveis para o correto manejo terapêutico e clínico desses pacientes. Dessa forma, o presente trabalho tem por objetivo avaliar a cardiotoxicidade induzida por quimioterápicos em pacientes atendidos em um sistema público em Santo André, comparando com dados existentes na literatura.

\section{MÉTODOS}

\section{DESENHO DO ESTUDO}

Estudo observacional retrospectivo com coleta de dados em sistema de prontuário eletrônico, realizado no Serviço de Oncologia vinculado a FMABC (Hospital Estadual Mário Covas, em Santo André). Foram considerados elegíveis para o estudo pacientes com diagnóstico confirmado de câncer de mama, em tratamento ou em seguimento, sendo tratadas com quimioterapia.

Critérios de exclusão foram pacientes que não realizaram tratamento quimioterápico ou que realizaram tratamento previamente a 2008, ano em que se iniciou o sistema de prontuário eletrônico no Hospital Estadual Mário Covas.

O estudo baseou-se na coleta de dados, através do levantamento de informações presentes nos prontuários, dos pacientes considerados elegíveis para o estudo. Com objetivo de avaliar a função cardíaca, avaliou-se o exame de ecocardiograma, feito de rotina para a maioria dos pacientes com câncer de mama que receberam medicação quimioterápica cardiotóxica. Para as pacientes em tratamento, avaliou-se o ecocardiograma antes do tratamento e o último ecocardiograma feito até o momento da análise; para pacientes em seguimento, avaliou-se o ecocardiograma antes do tratamento e o ecocardiograma feito ao final do tratamento. Dados incorretamente preenchidos foram excluídos da análise

\section{RESULTADOS}

Foram levantados dados de 311 pacientes com câncer de mama em diferentes estágios de tratamento quimioterápico. Desse total, $41,47 \%$ (129) realizaram ao menos um exame de ecocardiograma, e 13,50\% (42) fizeram 
dois ecocardiogramas. Apenas 8,03\% (25) dos pacientes realizaram ECO de modo adequado antes e depois do tratamento.

Alguns pacientes realizaram mais do que dois ecocardiogramas, mas não foram incluídos na análise devido aos seguintes motivos: realização do ECO fora do momento proposto pelo estudo (primeiro ECO realizado durante o tratamento ou segundo ECO realizado muito tardiamente após o término do tratamento); ausência do relato da fração de ejeção no laudo do exame, impossibilitando a comparação estatística.

As características sociodemográficas dos pacientes analisados podem ser observados na tabela 1.

Tabela 1: características sociodemográficas dos pacientes incluídos no estudo

\begin{tabular}{|c|c|c|c|c|}
\hline & & $\begin{array}{l}\text { Pacientes sem ECO } \\
\text { adequado }(n=286)\end{array}$ & $\begin{array}{l}\text { Pacientes com ECO adequado } \\
\qquad(n=25)\end{array}$ & Valor de $\mathrm{p}^{*}$ \\
\hline \multirow{2}{*}{ Idade } & Mediana & 57 & 59 & \multirow{2}{*}{-} \\
\hline & Variação & $25-92$ & $43-81$ & \\
\hline \multirow{5}{*}{ Raça } & Branca & $48,95 \%(140)$ & $48 \%(12)$ & \multirow{5}{*}{0,81} \\
\hline & Preta & $3,85 \%(11)$ & $8 \%(2)$ & \\
\hline & Amarela & $0,69 \%(2)$ & $0 \%(0)$ & \\
\hline & Parda & $22,02 \%(63)$ & $28 \%(7)$ & \\
\hline & Não encontrado & $24,47 \%(70)$ & $16 \%(4)$ & \\
\hline \multirow{4}{*}{ Tipo de neoplasia } & $\begin{array}{l}\text { Carcinoma Ductal } \\
\text { Invasivo }\end{array}$ & $77,62 \%(222)$ & $88 \%(22)$ & \multirow{4}{*}{0,32} \\
\hline & $\begin{array}{l}\text { Carcinoma Lobular } \\
\text { Invasivo }\end{array}$ & $8,39 \%(24)$ & $0 \%(0)$ & \\
\hline & Outros & $4,19 \%(12)$ & $4 \%(1)$ & \\
\hline & Não encontrado & $9,79 \%(28)$ & $8 \%(2)$ & \\
\hline \multirow{6}{*}{$\begin{array}{l}\text { Estágio de } \\
\text { tratamento }\end{array}$} & Neoadjuvante & $3,49 \%(10)$ & $4 \%(1)$ & \multirow{6}{*}{0,67} \\
\hline & Adjuvante & $17,83 \%(51)$ & $28 \%(7)$ & \\
\hline & Paliativo & $7,34 \%(21)$ & $12 \%(3)$ & \\
\hline & Seguimento & $35,66 \%(102)$ & $24 \%(6)$ & \\
\hline & Morto & $16,08 \%(46)$ & $16 \%(4)$ & \\
\hline & Não encontrado & $19,58 \%(56)$ & $16 \%(4)$ & \\
\hline \multirow{5}{*}{ Estadio clínico } & 1 & $10,83 \%(31)$ & $0 \%(0)$ & \multirow{5}{*}{0,21} \\
\hline & 2 & $28,67 \%(82)$ & $28 \%(7)$ & \\
\hline & 3 & $29,02 \%(83)$ & $24 \%(6)$ & \\
\hline & 4 & $20,62 \%(59)$ & $40 \%(10)$ & \\
\hline & Não encontrado & $10,83 \%(31)$ & $8 \%(2)$ & \\
\hline
\end{tabular}

*referente a teste Chi-quadrado

Os resultados do ECO podem ser encontrados na tabela 2. Avaliando os pacientes que realizaram quimioterapia e apresentavam o resultado de ECO adequadamente realizado antes e após a quimioterapia, observa-se diminuição média de 5,2\% (IC95\%: 2,31; 8,08. p=0,0005) da fração de ejeção após o fim do tratamento quimioterápico. 
Tabela 2: resultados da fração de ejeção em exame de ecocardiograma antes e após tratamento quimioterápico e comparação entre os valores.

\begin{tabular}{|c|c|c|c|c|}
\hline & & Média (\%) & Intervalo de confiança $95 \%$ & Valor de $\mathrm{p}^{*}$ \\
\hline \multirow{3}{*}{ Todos $(n=25)$} & ECO inicial & 67,12 & 63,$96 ; 70,27$ & \multirow{3}{*}{0,0005} \\
\hline & ECO final & 61,92 & 57,$42 ; 66,41$ & \\
\hline & Comparação & 5,2 & 2,$31 ; 8,08$ & \\
\hline \multirow{3}{*}{ Esquema AC-T ( $n=17)$} & ECO inicial & 70.11 & 68,$60 ; 71,62$ & \multirow{3}{*}{0,0043} \\
\hline & ECO final & 64.58 & 60,$34 ; 68 ; 83$ & \\
\hline & Comparação & 5.52 & 1,$61 ; 9,44$ & \\
\hline \multirow{3}{*}{$\begin{array}{l}\text { Esquema AC-T com } \\
\text { Trastuzumab }(n=11)\end{array}$} & ECO inicial & 70,36 & 68,$27 ; 72,45$ & \multirow{3}{*}{0,0292} \\
\hline & ECO final & 64,63 & 58,$16 ; 71,10$ & \\
\hline & Comparação & 5,72 & $-0,24 ; 11,69$ & \\
\hline \multirow{3}{*}{$\begin{array}{l}\text { Esquema AC-T sem } \\
\text { Trastuzumab }(n=6)\end{array}$} & ECO inicial & 69,66 & 66,$72 ; 72,61$ & \multirow{3}{*}{0,0300} \\
\hline & ECO final & 64,50 & 58,$46 ; 70,53$ & \\
\hline & Comparação & 5,15 & $-0,32 ; 10,65$ & \\
\hline
\end{tabular}

* referente a teste t de student pareado

Avaliando exclusivamente pacientes com ECO adequadamente realizado e cujo esquema quimioterápico utilizado correspondeu ao AC-T (Antraciclina e Ciclofosfamida seguida por Paclitaxel), observa-se diminuição média de 5,52\% (IC95\%: 1,61; 9,44. p=0,0043) da fração de ejeção após o fim do tratamento quimioterápico.

Dentre os pacientes que realizaram esquema AC-T, há aqueles com indicação para uso do trastuzumab (herceptin) e outros que não fizeram uso da medicação. Dentre os que fizeram uso do trastuzumab, foi observado diminuição da fração de ejeção de 5,72\% (IC95\%:-0,24; 11,69. $\mathrm{P}=0,0292$ ). Já para os que não fizeram seu uso, houve queda da fração de ejeção de 5,15\% (IC95\%: -0,32; 10,65. p=0,03).

\section{DISCUSSÃO}

Nas últimas décadas, o tratamento anti-neoplásico aprimorou-se consideravelmente, melhorando o prognóstico de pacientes oncológicos. ${ }^{3}$ Isso se evidencia especialmente em pacientes com câncer de mama, considerando que o uso da mamografia para rastreamento populacional possibilitou o diagnóstico precoce desse tipo de câncer, elevando a sobrevida em 5 anos para 89,7\% em casos adequadamente tratados. ${ }^{9}$

Apesar disso, o tratamento quimioterápico também trouxe importantes efeitos adversos, especialmente relacionados à cardiotoxicidade. ${ }^{3}$ Tais efeitos apresentam especial relevância no câncer de mama, considerando que estudos demonstraram maior suscetibilidade feminina à cardiotoxicidade, ${ }^{10} \mathrm{e}$ devido à elevada sobrevida em 5 anos, deixando mais evidente os efeitos clínicos da lesão cardíaca. ${ }^{9}$

Para o diagnóstico de cardiotoxicidade relacionada à quimioterapia, avalia-se a presença de sinais e sintomas relacionados à insuficiência cardíaca; redução global ou específica do septo intraventricular da fração de ejeção do ventrículo esquerdo; e redução de 5 a $55 \%$ da fração de ejeção basal do ventrículo esquerdo na presença de sinais ou sintomas de insuficiência cardíaca, ou redução de 10 a 55\% da fração de ejeção basal do ventrículo esquerdo na ausência de sinais ou sintomas. ${ }^{11,12}$ Importante ressaltar que a manifestação de cardiotoxicidade induzida por quimioterapia geralmente é subclínica, havendo disfunção sistólica ou diastólica que leva a insuficiência cardíaca irreversível, sendo fundamental a realização de exames que demonstrem a função cardíaca, não levando em consideração exclusivamente a clínica do paciente. ${ }^{11,13}$ 
Durante várias décadas, a cardiomiopatia induzida por terapêutica oncológica era quase exclusivamente associada ao uso de doses cumulativas de antraciclinas, que promovem lesões permanentes a nível celular. No entanto, o uso de novos agentes terapêuticos, como o anticorpo monoclonal Trastuzumab, induz uma disfunção transitória reversível dos miócitos sem que haja relação com a dose utilizada. ${ }^{3,4,7}$ Diversos estudos demonstraram efeitos tóxicos cardiovasculares associados ao uso dessa medicação. ${ }^{11,14,15}$ Dentre eles, uma revisão da literatura de Seidman et al. ${ }^{11}$ observou um aumento do risco de disfunção cardíaca associada ao trastuzumab, especialmente quando associado a antraciclina e ciclosfosfamida (esquema $A C$ ), sendo observado menor risco em pacientes recebendo trastuzumab exclusivo ou trastuzumab associado a paclitaxel.

Os anticorpos monoclonais como o Trastuzumab são muito utilizados no tratamento do câncer de mama, visto que 15 a $25 \%$ dos pacientes apresentam amplificação do gene HER2, onde atua o fármaco. ${ }^{4,16} \mathrm{O}$ uso de anticorpos monoclonais e antraciclinas concomitantes é frequente no tratamento do câncer de mama. Regimes de quimioterapia sem antraciclinas apresentam taxas menores de disfunção cardíaca quando comparados com regimes de uso combinado de trastuzumab e antraciclinas, 3-7\% e $27 \%$, respectivamente. ${ }^{14}$

Um dos principais fatores de risco cardiotóxicos associados ao uso antineoplásico de trastuzumab é a utilização de elevadas doses cumulativas de antraciclinas (> $300 \mathrm{mg} / \mathrm{m} 2$ ). Outros importantes fatores de risco incluem a disfunção ventricular esquerda associada ou não ao uso concomitante de antraciclinas, a pré-existência de hipertensão arterial sistêmica, um índice de massa corporal superior a 25 e a idade avançada dos doentes. Além disso, dados recentes demonstraram que doentes oncológicos idosos ( $\geq 70$ anos) com história de doença cardíaca e/ou diabetes apresentam um aumento da incidência de efeitos cardiotóxicos associados ao uso de trastuzumab no tratamento do câncer da mama. ${ }^{7}$

O presente estudo identificou uma relação estatisticamente significante quanto ao efeito cardiotóxico da quimioterapia, havendo queda média de $5 \%$ da fração de ejeção, especialmente relacionado ao esquema AC-T, que inclui o uso de antraciclinas e taxanos. Esse resultado é compatível com o estudo clínico de Sawaya et al., ${ }^{17}$ em que 81 mulheres diagnosticadas com câncer de mama HER2 positivo foram tratadas com antraciclinas seguido de taxanos e trastuzumab, sendo que o acompanhamento trimestral com ecocardiograma evidenciou queda de $5 \%$ da fração de ejeção ao longo de 15 meses, $p<0,0001$.

Apesar disso, dividindo-se os pacientes que realizaram o esquema AC-T entre aqueles que utilizaram o trastuzumab e os que não utilizaram, não foi possível observar uma queda da fração de ejeção com relação estatística significante (IC95\%:0,32; 10,65). Isso deve-se, provavelmente, ao baixo número de pacientes analisados no teste $\mathrm{t}$ de student pareado.

Assim, o desenvolvimento de estratégias para limitar a cardiotoxicidade apresenta fundamental importância, de forma a equilibrar os riscos cardiotóxicos com os benefícios da terapia anti-neoplásica em doentes oncológicos. Estas incluem a utilização de combinações otimizadas de quimioterapia, o encurtamento do tratamento e o acompanhamento rigoroso dos doentes tratados. ${ }^{10}$

Além da cardiotoxicidade induzida pela quimioterapia, no presente estudo foi identificado que apenas $13,50 \%$ dos pacientes com câncer de mama em tratamento quimioterápico realizaram ao menos dois exames de ecocardiograma. Isso evidencia um problema de saúde pública brasileiro, em que pacientes com câncer de mama realizam menos exames para rastreamento de disfunção cardíaca do que o necessário, o que propicia a manutenção do tratamento quimioterápico em pacientes que tiveram grande queda da fração de ejeção e cuja recomendação seria diminuição da dose do quimioterápico ou ajuste do esquema para prevenção da cardiotoxicidade.

Uma explicação para essa baixa realização de ECO por pacientes de risco seria a dificuldade de realização de exames no Sistema Único de Saúde (SUS), já evidenciada em estudos brasileiros como o de Spedo, ${ }^{18}$ em que há longa espera para realização do exame, de modo que o momento ideal para adequado diagnóstico de lesão cardíaca acaba sendo ultrapassado, mantendo-se o esquema quimioterápico em pacientes que estão apresentando disfunção cardíaca, levando a um pior prognóstico.

Para o diagnóstico precoce de disfunção cardíaca em pacientes com câncer de mama recebendo tratamento quimioterápico, estudos indicam a realização de exames a cada 3 meses. Isso pode ser observado no estudo clínico avaliando a cardiotoxicidade relacionada ao trastuzumab de Sawaya, ${ }^{17} \mathrm{em}$ que ecocardiograma e exames de sangue eram realizados a cada 3 meses por um total de 15 meses consecutivos, havendo cardiotoxicidade em $36 \%$ dos pacientes, sendo que apenas $6 \%$ apresentavam sintomas de insuficiência cardíaca. 
Apesar disso, em um estudo de Dores, ${ }^{19} 51$ pacientes com câncer de mama tratadas com trastuzumab foram avaliadas antes e 3 meses após o início do tratamento, não sendo observado diferença significante da fração de ejeção dos pacientes no estudo. Dessa forma, ainda há dúvida sobre a necessidade de realização de ecocardiograma a cada 3 meses para manejo adequado da cardiotoxicidade. Independentemente disso, o presente estudo evidenciou que menos de $15 \%$ das pacientes realizaram pelo menos dois exames ecocardiográficos ao longo do tratamento quimioterápico, indicando que faltam exames para rastreamento de disfunção cardíaca nessas pacientes, explicado pela dificuldade de realização de exames na realidade do sistema público de saúde brasileiro. ${ }^{18}$

Nesse âmbito, outros exames poderiam ser utilizados para diagnóstico de disfunção cardíaca induzida por quimioterapia. Em uma revisão sistemática da literatura de Dolci, ${ }^{13}$ foi identificado que a diminuição da fração de ejeção identificada em ecocardiograma apresenta baixa sensibilidade para diagnóstico de cardiomiopatias, enquanto que exames laboratoriais de troponina cardíaca podem apresentar valor preditivo negativo de $99 \%$ e permitem um diagnóstico eficiente de disfunção cardíaca até 3 meses antes das manifestações clínicas. Nesse mesmo estudo, afirma-se que faltam dados que confirmar a associação de peptídeos natriuréticos cardíacos para o diagnóstico de cardiomiopatia relacionada à quimioterapia.

Diversos estudos clínicos utilizam troponina ultrassensível I e fragmento $\mathrm{N}$-terminal do peptídeo natriurético tipo B como auxiliares ao ecocardiograma no diagnóstico precoce de cardiomiopatia induzida por quimioterapia. ${ }^{14,17}$ Considerando as dificuldades de realização de exames de custo mais elevado na realidade de saúde pública brasileira, como é o caso do ecocardiograma, a busca por exames mais baratos que permitam um adequado diagnóstico da miocardiopatia induzida por agentes quimioterápicos apresenta-se de fundamental relevância e pode vir a trazer importantes benefícios no prognóstico de pacientes oncológicos, especialmente pacientes com câncer de mama, que apresentam elevada taxa de sobrevida. ${ }^{9}$

Considerando os efeitos cardiotóxicos da quimioterapia, evidencia-se a necessidade de uma cooperação entre oncologistas e cardiologistas, devendo os primeiros estarem cientes da miocardiopatia induzida por quimioterapia de modo a prevenir esses efeitos; e os segundos preparados para auxiliarem oncologistas na avaliação dos pacientes baseado na escolha do tratamento anti-neoplásico. ${ }^{20}$

Trata-se de um estudo retrospectivo com o uso de dados secundários analisados em prontuários. Dessa forma, evidencia-se que uma parcela significativa de características sociodemográficas não foi encontrada, dificultando a avaliação da amostra utilizada no estudo.

Além disso, observa-se que menos de $15 \%$ das pacientes fizeram ao menos dois exames de ecocardiograma, e $8 \%$ puderam ser analisados. Dessa forma, há a possibilidade do estudo apresentar um viés de seleção, em que apenas pacientes que apresentavam maior risco de disfunção cardíaca ou que apresentaram maior toxicidade relacionada à quimioterapia realizaram novo exame ecocardiográfico, gerando um falso resultado de queda média da fração de ejeção de $5 \%$ relacionado ao tratamento. Apesar disso, os resultados encontrados estão condizentes com outros estudos na literatura. ${ }^{17}$

\section{CONCLUSÃO}

Nesse estudo, quimioterapia esteve relacionada à cardiotoxicidade e disfunção cardíaca em pacientes com câncer de mama, especialmente relacionado ao esquema associando antraciclinas e ciclofosfamida seguido de taxanos, assim como ao trastuzumab. Também foi identificado que menos de $15 \%$ realizaram pelo menos dois exames de ecocardiograma ao longo do tratamento quimioterápico, indicando deficiência do sistema público de saúde brasileiro em diagnóstico precoce de miocardiopatia induzida por agentes quimioterápicos.

\section{REFERÊNCIAS}

1. Brasil. Ministério da Saúde. Estimativas da incidência e mortalidade por câncer no Brasil [Internet]. Rio de Janeiro: Instituto Nacional de Câncer; 2016 [citado 2016 nov 3]. Disponível em: http://www2.inca.gov.br/wps/wcm/connect/tiposdecancer/site/home/mama

2. Brasil. Ministério da Saúde. Registro hospitalar de câncer - Relatório anual 1994/1998 [Internet]. Rio de Janeiro: Instituto Nacional de Câncer; 2016 [citado 2016 nov 3]. Disponível em: http://www.inca.gov.br/rhc/docs/INCA_94_98.pdf 
3. Minami M, Matsumoto S, Horiuchi H. Cardiovascular sideeffects of modern cancer therapy. Circ J. 2010;74(9):1779-86. http://dx.doi.org/10.1253/circj.CJ-10-0632. PMid:20716834.

4. Adão R, Keulenaer G, Leite-Moreira A, Brás-Silva C. Cardiotoxicidade associada à terapêutica oncológica: mecanismos fisiopatológicos e estratégias de prevenção. Rev Port Cardiol. 2013;32(5):395-409.

http://dx.doi.org/10.1016/j.repc.2012.11.002. PMid:23623503.

5. Karanth NV, Roy A, Joseph M, Pasquale C, Karapetis C, Koczwara B. Utility of prechemotherapy echocardiographical assessment of cardiac abnormalities. Support Care Cancer. 2011;19(12):2021-6. http://dx.doi.org/10.1007/s00520-0101054-z. PMid:21120541.

6. Mina A, Rafei H, Khalil M, Hassoun Y, Nasser Z, Tfayli A. Role of baseline echocardiography prior to initiation of anthracycline-based chemotherapy in breast cancer patients. BMC Cancer. 2015;15(1):10.

http://dx.doi.org/10.1186/s12885-014-1004-0. PMid:25605569.

7. Kalil R Fo, Hajjar LA, Bacal F, Hoff PM, Diz MP, Galas FRBG. Diretriz brasileira de cardio-oncologia da sociedade brasileira de cardiologia. Arq Bras Cardiol. 2011;96(2 Suppl 1):1-52. http://dx.doi.org/10.1590/S0066782X2011000700001.

8. Sá MPBO, Gomes RAF, Silva NPC, Sá MVBO, Calado I Fo. Cardiotoxicity and chemotherapy. Rev Bras Clin Med. $2009 ; 7(5): 326-30$.

9. Young JL Jr, Roffers SD, Ries LAG, et al., editors. SEER summary staging manual - 2000 codes and coding instructions. Bethesda: National Cancer Institute; 2001 [citado 2016 nov 3]. Disponível em: https://seer.cancer.gov/tools/ssm/ssm2000/SSSM2000-122012.pdf

10. Doyle JJ, Neugut Al, Jacobson JS, Grann VR, Hershman DL. Chemotherapy and cardiotoxicity in older breast cancer patients: a population-based study. J Clin Oncol. 2005;23(34): 8597. PMid:16314622.

11. Seidman A, Hudis C, Pierri MK, et al. Cardiac dysfunction in the trastuzumab clinical trials experience. J Clin Oncol. 2002;20(5):1215-21. http://dx.doi.org/10.1200/JCO.2002.20.5.1215. PMid:11870163.

12. Florescu M, Cinteza M, Vinereanu D. Chemotherapy-induced cardiotoxicity. Maedica (Buchar). 2013;8(1):59-67. PMid:24023601.

13. Dolci A, Dominici R, Cardinale D, Sandri MT, Panteghini M. Biochemical markers for prediction of chemotherapyinduced cardiotoxicity: systematic review of the literature and recommendations for use. Am J Clin Pathol. 2008;130(5):688-95. http://dx.doi.org/10.1309/AJCPB66LRIIVMQDR. PMid:18854260.

14. Fiúza M. Cardiotoxicity associated with trastuzumab treatment of HER2+ breast cancer. Adv Ther. 2009;26(Suppl 1):917. http://dx.doi.org/10.1007/s12325-009-0048-z. PMid:19669637.

15. Onitilo AA, Engel JM, Stankowski RV. Cardiovascular toxicity associated with adjuvant trastuzumab therapy: prevalence, patient characteristics, and risk factors. Ther Adv Drug Saf. 2014;5(4):154-166.

http://dx.doi.org/10.1177/2042098614529603.

16. Gianni L, Salvatorelli E, Minotti G. Anthracycline cardiotoxicity in breast cancer patients: synergism with trastuzumab and taxanes. Cardiovasc Toxicol. 2007;7(2):67-71. http://dx.doi.org/10.1007/s12012-007-0013-5. PMid:17652806.

17. Sawaya H, Sebag IA, Plana JC, et al. Assessment of echocardiography and biomarkers for the extended prediction of cardiotoxicity in patients treated with anthracyclines, taxanes, and trastuzumab. Circ Cardiovasc Imaging. 2012;5(5):596603. http://dx.doi.org/10.1161/CIRCIMAGING.112.973321. PMid:22744937.

18. Spedo SM, Pinto NRS, Tanaka OY. O difícil acesso a serviços de média complexidade do SUS: o caso da cidade de São Paulo, Brasil. Physis. 2010;20(3):953-72. http://dx.doi.org/10.1590/S0103-73312010000300014.

19. Dores H, Abecasis J, Correia MJ, et al. Detection of early sub-clinical trastuzumab-induced cardiotoxicity in breast cancer patients. Arq Bras Cardiol. 2013;100(4):328-32. PMid:23525273. 
20. Albini A, Pennesi G, Donatelli F, Cammarota R, Flora S, Noonan DM. Cardiotoxicity of Anticancer Drugs: The Need for Cardio-Oncology and Cardio-Oncological Prevention. JNCI Journal of the National Cancer Institute. 2010;102(1):1-11. http://dx.doi.org/10.1093/jnci/djp440. PMid:20007921.

Autor correspondente: Igor Luiz Argani

E-mail: igorlargani@gmail.com

Rua Rio Grande do Sul, 681 - São Caetano do Sul, Brasil. CEP: 09510-0

Tel: (11) 96477-5536 